\title{
Taxa de Juros e Default em Mercados de Empréstimos Colateralizados
}

\section{Sergio Ricardo Faustino Batista - José Angelo Divino - Jaime Orrillo}

Submetido: 26 de março de 2011; aceito para publicação: 23 de setembro de 2011

Resumo: Este artigo investiga como variações nas taxas de juros afetam a probabilidade de default (PD) em um modelo de equilíbrio geral com mercados incompletos e exigência de colateral. Teoricamente, a PD possui relação positiva com taxa de juro real do empréstimo e negativa com a taxa de juro real da economia. Empiricamente, essas relações são confirmadas por meio da estimação do modelo de risco proporcional de Cox para uma grande amostra de empréstimos colateralizados. Dentre as variáveis de controle, há características do indivíduo, do contrato e da economia como um todo. Intuitivamente, uma taxa de juro real básica mais baixa implica menor retorno das operações de tesouraria, levando os bancos a ampliarem suas carteiras de crédito, emprestando para indivíduos mais arriscados.

Palavras-Chave: probabilidade de default, mercados incompletos, análise de sobrevivência

Abstract: This paper investigates how changes in interest rates affect the probability of default (PD) in a general equilibrium model with incomplete markets and collateral requirement. Theoretically, the

José A. Divino e Jaime Orrillo, agradecem ao CNPq pelo apoio financeiro. Os autores agradecem a dois pareceristas anônimos pelos valiosos comentários e sugestões. Todos os erros remanecentes são responsabilidade exclusiva dos autores.

Sergio Ricardo Faustino Batista

Mestrado e Doutorado em Economia da Universidade Católica de Brasília - Endereço para contato: SGAN 916, sala A-113 - CEP: 70.719-160 Brasília-DF - E-mail: sergio.batista@caixa.gov.br José Angelo Divino

Mestrado e Doutorado em Economia da Universidade Católica de Brasília - Endereço para contato: SGAN 916, sala A-116 - CEP: 70.719-160 Brasília - DF - E-mail: jangelo@pos.ucb.br Jaime Orrillo

Mestrado e Doutorado em Economia da Universidade Católica de Brasília - Endereço para contato: SGAN 916, sala A-115 - CEP: 70.719-160 Brasília - DF - E-mail: orrillo@pos.ucb.br 
PD has a positive relationship with the loan real interest rate and negative with the economy real interest rate. Empirically, those relationships are confirmed by the estimation of the Cox proportional hazard model for a large sample of collateralized loans. Among the control variables, there are characteristics of the individuals, contracts, and economy as a whole. Intuitively, a lower real interest rate reduces earnings from financial operations, leading the banks to increase their credit portfolios by lending for riskier individuals.

Keywords: default probability, incomplete markets, survival analysis

\section{JEL Classification: D52, C81}

\section{Introdução}

A recente crise financeira internacional, que se iniciou nos Estados Unidos ao final de 2007 e, rapidamente, contagiou outros países, tornou patente que diversos fatores podem afetar a capacidade de pagamento dos tomadores de crédito, tendo como consequência a elevação dos níveis de inadimplência (ou default) no mercado. Podem-se destacar, por exemplo, condições macroeconômicas do país, características individuais dos tomadores de crédito e dos contratos de empréstimo assumidos. Nesse sentido, variáveis como taxa de desemprego, taxa de juros da economia, taxa de juros do contrato e o valor da dívida assumida podem influenciar a probabilidade de default de determinado indivíduo. É importante identificar os determinantes dessa probabilidade não apenas para atender as regras do acordo de Basiléia, que prevê o uso de modelos de gerenciamento de risco por instituições financeiras, ${ }^{1}$ mas também para orientar a edição de medidas de política econômica que impactam sobre o mercado financeiro.

O objetivo deste artigo é investigar a relação entre taxas de juros e probabilidade de default na economia brasileira. Para tanto, desenvolve-se uma modelagem teórica com base em um modelo de equilíbrio geral com mercados incompletos, exigência de garantia (ou colateral) e punição aos indivíduos em caso de default. A referência básica é o

\footnotetext{
${ }^{1}$ O comunicado 19.028 do Banco Central do Brasil, de 29/10/2009, apresenta o cronograma de implantação das regras estabelecidas no acordo de Basiléia.
} 
modelo proposto por Dubey, Geanakoplos e Zame (1995), que trata de uma economia com venda de ativos garantida por bens duráveis, penalidade não pecuniária na função utilidade, perda do colateral em caso de default e obrigação de amortizar a dívida ao longo do tempo. Os resultados teóricos são testados empiricamente por meio da aplicação do modelo de risco proporcional de Cox com variáveis dependentes do tempo a uma amostra de empréstimos colateralizados obtida junto a um grande banco brasileiro.

A escolha dessa estratégia de modelagem deve-se à sua adequação ao segmento imobiliário do mercado financeiro brasileiro, onde há aplicação de penalidade ao agente inadimplente e exigência de garantia, real ou financeira, para assegurar o empréstimo. Pascoa e Seghir (2009) também adotaram a estratégia de incluir, simultaneamente, penalidade não pecuniária e colateral na modelagem de default. Além disso, teoricamente, a existência de equilíbrio já está demonstrada na literatura, mesmo com a ocorrência de default.

Operações imobiliárias são exemplos mais comuns de empréstimos colateralizados. No passado, essas operações eram realizadas por instituições criadas para essa finalidade que, em razão de suas características, assumiam problemas de seleção adversa e incorriam na concentração de investimentos, com concessão de crédito realizada com base na comprovação de renda. Mais recentemente, essas operações passaram a ser realizadas por outros tipos de instituições financeiras, tais como bancos múltiplos e comerciais com portfólio diversificado, e com o uso de ferramentas de avaliação de risco mais sofisticadas.

$\mathrm{Na}$ literatura teórica sobre modelos de equilíbrio geral com mercados incompletos e possibilidade de default, destacam-se os trabalhos de Dubey, Geanakoplos e Shubik (1990) e Zame (1993). O primeiro propõe a extensão da modelagem de equilíbrio geral com mercados incompletos para incluir a ocorrência de default. Já o segundo atribui ao default a elevação da eficiência econômica, pois permite a entrada no mercado de indivíduos que possuem elevada probabilidade, mas com certo grau de incerteza de não honrar os compromissos financeiros assumidos. Por sua vez, Dubey, Geanakoplos e Zame (1995) introduziram a exigência de colateral e mantiveram a premissa de penalidade na função utilidade do indivíduo em caso de default. 
Empiricamente, desde o trabalho pioneiro de Barth et al. (1983), que tratou o crédito pessoal com microdados, a modelagem de default também vem ganhando espaço na literatura. Ali e Daly (2010), por exemplo, avaliaram o impacto de variáveis macroeconômicas e sociodemográficas na demanda por produtos financeiros. No caso brasileiro, Martins e Galli (2007) aplicaram análise de sobrevivência para identificar antecipadamente o risco de concordata de empresas de capital aberto que operam na Bolsa de Valores de São Paulo. Divino, Lima e Orrillo (2010) analisaram, teórica e empiricamente, a relação entre taxas de juros e probabilidade de default para empréstimos sem garantia. Já Chu (2001) identifica os determinantes macroeconômicos do risco de crédito para o Brasil, usando dados agregados de séries temporais. Especificamente para o mercado imobiliário, pode-se destacar Lin e White (2001), que analisaram a relação entre isenção de falência e disponibilidade de crédito. Outros trabalhos empíricos importantes, aplicados a diferentes países e usando técnicas econométricas diversas, incluem Bellotti e Crook (2007), Carling et al. (2007), Banasik et al. (1999), Tang et al. (2007), Stepanova e Thomas (2001, 2002) e Andreeva (2006).

As contribuições deste artigo são tanto teóricas quanto empíricas. Inicialmente, demonstra-se a existência de uma relação teórica negativa entre a taxa de juros real da economia e a probabilidade de default, e positiva entre a taxa de juro real do empréstimo e a probabilidade de default. Então, aplica-se o modelo de risco proporcional de Cox a uma amostra de 637.704 empréstimos colateralizados de uma carteira imobiliária de um grande banco brasileiro no período de janeiro de 1997 a agosto de 2009. Os resultados confirmam os sinais encontrados para a relação entre taxas de juros e probabilidade de default pelo modelo teórico.

Intuitivamente, tem-se que uma redução da taxa de juros decorrente de uma política monetária expansionista, por exemplo, implica menor retorno financeiro nas operações de tesouraria realizadas por instituições financeiras. Essa perda de receita é compensada por meio da expansão da carteira de crédito. Para tanto, há uma flexibilização da avaliação de risco na concessão de crédito, tendo como consequência a elevação da probabilidade de default. Esta situação fica bem evidenciada em períodos de crise econômica, como aquela que teve origem no mercado hipotecário americano ao final de 2008 e se espalhou, em maior ou menor intensidade, por diversos países, 
incluindo o Brasil. As medidas adotadas para debelar a crise, que incluíam expansão do crédito, acabaram por elevar a probabilidade de default. Já uma elevação na taxa de juro real do contrato aumenta a probabilidade de default porque reduz a capacidade de pagamento do tomador.

O artigo está organizado da seguinte forma. A próxima seção apresenta o modelo de equilíbrio geral com mercados incompletos, default, colateral e penalidade na utilidade, usado na derivação da relação entre taxas de juros e probabilidade de default. A terceira seção trata do procedimento econométrico utilizado, representado pelo modelo de risco proporcional de Cox com covariáveis dependentes do tempo. A quarta seção apresenta os dados e os resultados da evidência empírica. Por fim, a quinta seção é dedicada às observações conclusivas.

\section{Modelo Teórico}

O modelo é uma versão simplificada de Dubey, Geanakoplos e Zame (1995), que modelam uma economia com mercados financeiros incompletos onde a venda de ativos é colateralizada por bens duráveis. A simplificação ocorre ao estilo de Magill e Quinzii (1996), permitindo nos concentrar apenas no equilíbrio financeiro. Em caso de inadimplência, os tomadores perdem o colateral, que é repartido entre os emprestadores. Além de perder o colateral, os tomadores inadimplentes sofrem uma penalidade diretamente em termos de utilidade, que os obriga a pagar mais do que o mínimo entre a dívida corrigida pelos juros ${ }^{2}$ e o colateral depreciado.

O modelo tem 2 períodos, com incerteza somente no segundo período, o qual é modelado por um conjunto finito de estados da natureza $\mathcal{S}=\{1, \ldots, S\}$. Há um único bem em cada período e em cada estado da natureza de maneira que o conjunto de consumo é $R_{+}^{S+1}$.

O conjunto de ativos financeiros é $\mathcal{J}=\{1, \ldots, J\}$. Cada ativo $j \in \mathcal{J}$ é representado pelo vetor $r^{j} \in R_{+}^{S}$, onde $r^{1}=(1, \ldots, 1)$ é assumido ser $\mathrm{o}$ ativo livre de risco de mercado que paga uma unidade da mercadoria em cada estado da natureza. Assume-se, também, que existe

\footnotetext{
${ }^{2}$ Isto para o caso de empréstimos financeiros. No caso de ações, a comparação é feita entre o rendimento futuro e o colateral depreciado.
} 
um único ativo arriscado sobre o qual os agentes podem inadimplir. Assim, há $J=2$ ativos no mercado. Esses ativos disponíveis para transações podem ser interpretados como dívida do governo e empréstimos sujeitos ao risco de crédito. Os preços desses ativos são $q$ e $\pi$, respectivamente. Por hipótese, os mercados são incompletos, isto é $S>2$.

Denotemos por $C$ o valor da garantia por cada unidade do ativo arriscado vendido no primeiro período. A garantia é durável e seu valor é contingente ao estado da natureza do segundo período. Assim o seu valor futuro é representado pelo vetor $C=\left(C_{1}, \ldots C_{S}\right) \in R_{++}^{S}$.

Cada indivíduo $h \in \mathcal{H}=\{1, \ldots, H\}$ caracteriza-se pela função de utilidade, $u^{h}: R_{+}^{S+1} \rightarrow R$, assumida ser duas vezes diferenciável, estritamente crescente e côncava e pelo vetor de suas dotações inicias $\omega^{h} \in R_{++}^{S+1}$. Além disso, cada indivíduo $h$ sofre uma penalidade comum em sua utilidade, $\lambda \in R_{+}^{S}$, que é proporcional à quantia não paga do empréstimo. Por essa razão, o plano de entregas $D_{s}$ satisfaz à seguinte desigualdade: ${ }^{3}$

$$
0<\min \left\{r_{s}, C_{s}\right\} \varphi \leq D_{s} \leq r_{s} \varphi
$$

onde $\varphi$ é a quantidade de ativos vendidos. ${ }^{4}$

\subsection{Problema do Indivíduo}

O indivíduo escolhe um vetor $(x, \theta, \varphi, b, D)$ que consiste em consumo $(x)$, investimento em ativos arriscados $(\theta, \varphi)$, sendo $\theta$ a quantidade comprada e $\varphi$ a quantidade vendida do ativo arriscado, investimento em ativo sem risco $(b)$ e um plano de entregas $(D)$ com o objetivo de maximizar o seu payoff dado por:

$$
V^{h}(x, \theta, \varphi, b, D)=u^{h}\left(x_{o}+C \varphi, \tilde{x}\right)-\sum_{s} \lambda_{s}\left[r_{s} \varphi-D_{s}\right]^{+}
$$

\footnotetext{
${ }^{3}$ Plano de entregas refere-se ao montante da dívida total (empréstimo mais juros) que será pago ao credor. Como há default no modelo, esse plano de entregas está entre 0 (default total) e o montante total da dívida.

${ }^{4}$ Pascoa e Seghir (2009) também modelam, simultaneamente, penalidade e colateral no problema do consumidor.
} 
sujeito às seguintes restrições:

$$
\begin{aligned}
& x_{o}+q b+\pi \theta+C \varphi \leq \omega_{o}^{h}+\pi \varphi \\
& x_{s}+D_{s} \leq \omega_{s}^{h}+t_{s} r_{s} \theta+(1+r) b+C_{s} \varphi, s \in \mathcal{S} \\
& 0<\min \left\{r_{s}, C_{s}\right\} \varphi \leq D_{s}, s \in \mathcal{S}
\end{aligned}
$$

A Equação (1) mostra que o payoff do indivíduo é a diferença entre a utilidade do consumo nos 2 períodos e a penalidade proporcional ao montante de dívida não paga. $\mathrm{O}$ parâmetro que representa a penalidade, $\lambda_{s}$, pode ser dependente do estado da natureza. A variável aleatória $\tilde{x}$ é o consumo dependente do estado no segundo período.

As Equações (2) e (3) representam restrições orçamentárias usuais. A restrição (2) estabelece que consumo, investimentos em ativos arriscados e sem risco e aquisição de colateral realizados no primeiro período são financiados por dotação inicial e empréstimos contraídos no período inicial.

Já a restrição (3) define que, em cada estado da natureza no segundo período, consumo e pagamento de empréstimo são financiados por valor da dotação, retornos financeiros de investimentos em ativo sem risco e em empréstimos privados que não inadimpliram e valor do colateral depreciado.

Por fim, a condição (4) impõe que a quantidade de default pode variar entre zero, quando há pagamento total da dívida, e o total da dívida, quando não há qualquer reembolso do empréstimo.

Note-se que apenas (2) e (3) são restrições orçamentárias. No entanto, a condição (4) satisfaz o plano de entregas devido à penalidade que o indivíduo sofre em sua função utilidade.

\subsection{Equilibrio}

Um equilíbrio para esta economia consiste de uma taxa de pagamento $t \in[0,1]^{S}$, um vetor de preços $(q, \pi) \in R_{+}^{2}$, um plano de consumo-in- 
vestimento $\left[x^{h}, b^{h},\left(\theta^{h}, \varphi^{h}\right)\right]_{h \in \mathcal{H}} \in \mathrm{R}_{+}^{(\mathrm{S}+1) \mathrm{H}} \times \mathrm{R}^{\mathrm{H}} \times \mathrm{R}_{+}^{2 \mathrm{H}}$ e um plano de entregas $\left(D^{h}\right)_{h \in \mathcal{H}} \in R_{+}^{S H}$, satisfazendo as seguintes propriedades:

1. As escolhas são ótimas. Isto é, para cada $h \in \mathcal{H}$ o vetor $\left(x^{h} ; b^{h} ;\left(\theta^{h}, \varphi\right)^{h} ; D^{h}\right)$ maximiza o payoff $(1)$ sujeito às restrições (2), (3) e (4).

2. Mercado de ativos está equilibrado:

$$
\sum_{h} \theta^{h}=\sum_{h} \varphi^{h}, \quad \sum_{h} b=0
$$

3. A taxa de pagamento:

$$
t_{s}=\frac{\sum_{h} D_{s}^{h}}{\sum_{h} r_{s} \varphi^{h}} \text {, desde que } \sum_{h} \varphi^{h}>0,
$$

é racionalmente antecipada, implicando que a taxa de inadimplência, $k_{s}=1-t_{s}$, também o seja. Observe que os itens 2 e 3 implicam que o mercado de bens também esteja equilibrado. Isto é,

$$
\begin{aligned}
& \sum_{h}\left(x_{o}^{h}+C \varphi^{h}\right)=\sum_{h} \omega_{o}^{h} \\
& \sum_{h} x_{s}^{h}=\sum_{h}\left(\omega_{o}^{h}+C_{s} \varphi^{h}\right), s \in \mathcal{S}
\end{aligned}
$$

O resultado a seguir é devido a Dubey, Geanakoplos e Zame (1995).

Teorema 1 Dubey, Geanakoplos e Zame (1995): Sob as hipóteses usuais sobre as utilidades e dotações iniciais, sempre existe um equilíbrio para a economia com colateral independentemente da natureza dos ativos, sejam eles reais ou nominais.

A aplicação desse teorema, demonstrado em Dubey, Geanakoplos e Zame (1995), garante a existência de equilíbrio no modelo discutido na seção anterior. A nossa principal contribuição teórica é reportada no teorema a seguir. 
Teorema 2 Para cada estado $s \in \mathcal{S}$ em equilíbrio, as desigualdades seguintes são verdadeiras:

$$
\frac{\partial k_{s}}{\partial R_{s}} \geq 0 e \frac{\partial k_{s}}{\partial R}<0
$$

em que $R_{s}=\frac{r_{s}}{\pi}$ é a taxa de retorno real do ativo arriscado e $R=\frac{1+r}{q}$ é a taxa de retorno real do ativo livre de risco.

A demonstração usa as condições de primeira ordem do problema do indivíduo e o teorema da função implícita, sendo apresentada anexa.

A primeira desigualdade estabelece que a taxa de default depende positivamente da taxa de juro real do empréstimo colateralizado. Interpretando o ativo sem risco como um título do governo, a segunda desigualdade indica que a taxa de default depende negativamente da taxa de juro real dos títulos públicos. No caso brasileiro, a taxa paga pelos títulos públicos é representada pela taxa de juro do over selic, que também é o instrumento da política monetária de metas de inflação. Na evidência empírica, para ambas as desigualdades, a taxa de juro real é obtida a partir da taxa bruta de juro descontada da taxa de inflação. ${ }^{5}$ As taxas de juros são expressas em termos reais porque não há moeda no modelo, sendo que o bem de consumo é o numerário.

Intuitivamente, a primeira desigualdade indica que uma taxa de juro do empréstimo mais alta aumenta a probabilidade de default porque reduz a capacidade de pagamento do tomador do empréstimo. Por outro lado, a segunda desigualdade sugere que reduções na taxa de juro real básica da economia, por causa de uma política monetária expansionista, por exemplo, implicam menor retorno financeiro nas operações de tesouraria realizadas por instituições financeiras. Para compensar essa perda de receita, os bancos relaxam as análises de risco, concedendo crédito a tomadores mais arriscados e, consequentemente, elevando a probabilidade de default na economia. Note que, conforme mostram Dubey, Geanakoplos e Shubik (2005), seleção adversa é eliminada do modelo pelo requerimento de colateral e, portanto, não afeta o segundo resultado do Teorema 2 .

\footnotetext{
${ }^{5}$ Sendo i a taxa de juro e $\pi$ a taxa de inflação, temos que $\left(1+i_{\text {real }}\right)=\left(1+i_{\text {bruta }}\right) /(1+\pi)$.
} 
A próxima seção descreve a modelagem econométrica usada para testar empiricamente as relações teóricas entre as taxas de juros e a probabilidade de default na economia brasileira.

\section{Modelo Econométrico}

A análise de sobrevivência é usada na modelagem porque permite estudar a duração de um evento até a ocorrência de falha em determinada população, facilita a inclusão de observações que não falharam no período, chamadas censuradas, e possibilita incluir variáveis macroeconômicas dependentes do tempo. Quando um indivíduo toma um empréstimo financeiro em uma instituição bancária, por contrato, é obrigado a realizar pagamentos frequentes, geralmente mensais, até a quitação da dívida. Aqueles que deixaram de pagar determinado número de parcelas são considerados inadimplentes. Assim, defina:

$D(I, t)=\left\{\begin{array}{l}1, \text { caso os dias de atraso do tomador i sejam } t \geq \alpha \\ 0, \text { caso os dias de atraso do tomador } i \text { sejam } t<\alpha\end{array}\right\}$

em que $i$ refere-se ao $i$-ésimo cliente de determinada carteira de crédito e $t$ é o tempo medido em dias. O número de dias em atraso, $\alpha$, que caracteriza um cliente como inadimplente, é determinado pela matriz de migração ao atraso. Essa matriz calcula a probabilidade de migração de um dado nível de atraso para um nível de atraso superior. Na realidade, isso é uma probabilidade condicional, em que o próximo estado depende exclusivamente do estado corrente. Por causa da estrutura de nossa amostra, o intervalo de atraso na matriz de migração é 30 dias, o qual define a frequência de pagamento das parcelas do empréstimo.

O ponto de corte definindo default, por norma interna da instituição financeira detentora do banco de dados, é de 180 dias. No caso de empréstimos imobiliários, essa definição coincide com a que é usada pelo mercado. Na matriz de migração reportada na Tabela 1, está localizada na faixa em que a probabilidade de migrar para um nível superior de atraso é de $80 \%$. Esse ponto de corte assegura que a grande maioria dos clientes que atinge essa faixa de atraso não deixará o estado de default, resultando em uma perda financeira para a instituição. Observe que um cliente pode estar inadimplente em um 
mês e deixar essa condição no próximo, desde que pague pelo menos uma parte das parcelas em atraso. Contudo, isso acontece somente em $20 \%$ dos casos sob a classificação adotada de default.

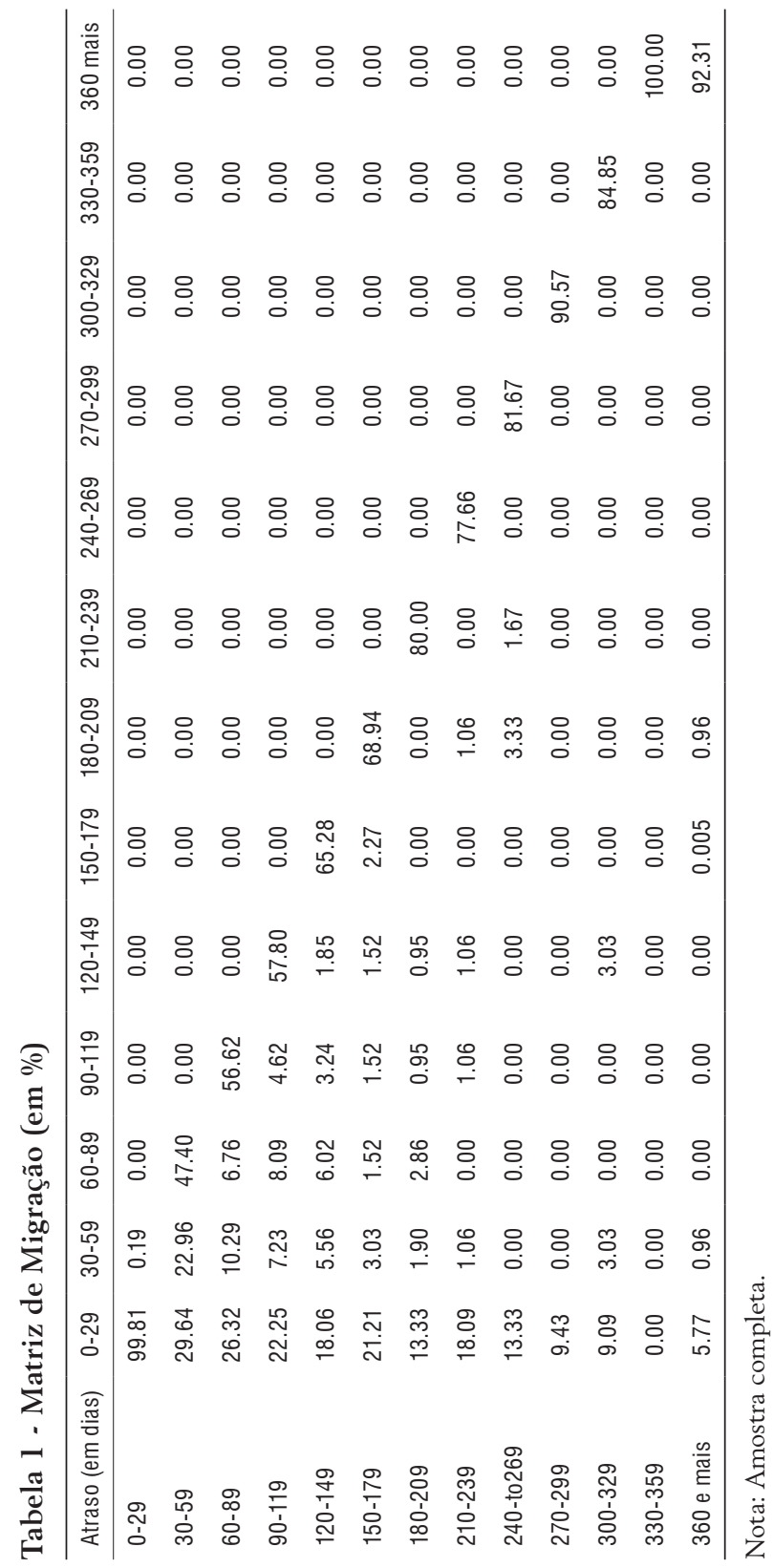




\subsection{Modelo de Risco Proporcional de Cox}

Dentre os modelos econométricos que podem ser usados para se analisar probabilidade de default há técnicas não paramétricas, modelos probabilísticos, regressões logísticas e análise de sobrevivência. Esta última foi escolhida porque representa bem o processo de empréstimo-inadimplência, permite modelar ambos, probabilidade e tempo de default, fornece previsões como função do tempo e permite a inclusão de covariáveis dependentes do tempo. Essa última característica surge como uma modificação ao modelo de risco proporcional de Cox $(1972,1975)$. Outro aspecto importante é sua versatilidade, dado que não assume nenhuma distribuição de probabilidade para os dados.

A função de risco do modelo de Cox é dada por:

$$
h(t)=\lim _{\delta \rightarrow 0}\left(\frac{P(t \leq T<t+\delta t \mid T \geq t}{\delta t}\right)
$$

e o modelo de risco proporcional de Cox com covariáveis dependentes do tempo pode ser escrito como:

$$
h(t, X(t), \beta)=h_{o}(t) g\left(X^{\prime}(t) \beta\right)
$$

em que $g($.$) é uma função não negativa que deve ser especificada, tal$ que $g(0)=1, X(t)$ é um vetor de covariáveis, $\beta$ é um vetor de parâmetros a ser estimado, e $h_{o}(t)$ é uma função não paramétrica de risco que depende do tempo mas não das covariáveis. A função $g\left(X^{\prime}(t) \beta\right)$ é o componente paramétrico e, usualmente, assume a forma:

$g\left(X^{\prime}(t) \beta\right)=\exp \left\{X^{\prime}(t) \beta\right\}=\exp \left\{\beta_{1} x_{1}+\ldots+\beta_{n} x_{n}\right\}$

O modelo é conhecido como de risco proporcional porque a taxa de falha de quaisquer dois indivíduos é constante no tempo. Essa hipótese é quebrada quando o modelo inclui covariáveis dependentes do tempo. A interpretação dos coeficientes estimados, $\beta$, também é afetada. Cada coeficiente estimado pode ser visto como o logaritmo 
da função de risco quando o valor da covariável sob análise muda em uma unidade e todas as demais são mantidas constantes.

A probabilidade de sobrevivência até o período $t$ é:

$$
S(t)=P(t \leq T)=\exp \left(-\int_{0}^{t} h(u) d u\right)
$$

Aqui, $S(t)$ representa a probabilidade que o indivíduo não entre em default até o período t. Assim, podemos considerar:

$$
S(t)=1-P D(t)
$$

em que $P D(t)$ é a probabilidade de default no período $t$.

Os indivíduos que não entraram em default durante o período são classificados como censurados e seu tempo de sobrevivência é o último período em que foram observados. Assim,

$$
c_{i}=\left\{\begin{array}{l}
0, \text { se a observação i é censurada } \\
1, \text { caso contrário }
\end{array}\right\}
$$

Indivíduos que, por algum motivo, não forem observados também são considerados censurados e recebem o mesmo tratamento.

A estimação dos parâmetros do modelo é dificultada pela presença do componente não paramétrico. Cox propôs um método de máxima verossimilhança parcial, que considera o conhecimento da história passada de falhas na construção da função de máxima verossimilhança. A ideia geral do método é descrita a seguir.

Assuma que, para uma amostra de $n$ indivíduos, haja $k \leq n$ falhas distintas nos períodos $t_{1}<t_{2}<\ldots<t_{k}$. A probabilidade condicional que a $i$-ésima observação falhará no tempo $t_{i}$, dadas as observações que estão sob risco em $t_{i}$, é: 


$$
\begin{aligned}
& P\left[i \in I \text { falhe em } t_{i} \mid \text { uma falha em } t_{i} \text { e a história até } t_{i}\right] \\
& =\frac{h_{i}\left(t \mid X_{i}\right)}{\sum_{j \in R\left(t_{i}\right)} h_{j}\left(t \mid X_{j}\right)}=\frac{h_{o} \exp \left(X_{i}^{\prime}(t) \beta\right)}{\sum_{j \in R\left(t_{i}\right)} h_{o} \exp \left(X_{j}^{\prime}(t) \beta\right)}=\frac{\exp \left(X_{i}^{\prime}(t) \beta\right)}{\sum_{j \in R\left(t_{i}\right)} \exp \left(X_{j}^{\prime}(t) \beta\right)}
\end{aligned}
$$

em que $R\left(t_{i}\right)$ é um conjunto de índices de observações que estão sob risco no período $t_{i}$, isto é, são observações que não apresentaram falha até o tempo $t_{i}$. Assim, o termo $h_{o}(t)$ desaparece. Para estimar os parâmetros do modelo, a função de máxima verossimilhança é dada pelo produto de todos os termos representados por (12) associados a distintos tempos de falha. Isto é,

$$
L(\beta)=\prod_{i=1}^{k} \frac{\exp \left(X_{i}^{\prime}(t) \beta\right)}{\sum_{j \in R\left(t_{i}\right)} \exp \left(X_{j}^{\prime}(t) \beta\right)}=\Pi_{i=1}^{n}\left(\frac{\exp \left(X_{i}^{\prime}(t) \beta\right)}{\sum_{j \in R\left(t_{i}\right)} \exp \left(X_{j}^{\prime}(t) \beta\right)}\right)^{c_{i}}
$$

em que $c_{i}$ é o indicador de censura. Para encontrar valores de $\beta$ que maximizam a função de máxima verossimilhança parcial, $L(\beta)$, deve-se resolver o sistema de equações definido por $U(\beta)=0$, no qual $U(\beta)$ é o vetor de scores de $l(\beta)=\log (L(\beta))$. Assim,

$$
U(\beta)=\sum_{i=1}^{n} c_{i}\left[X_{i}-\frac{\sum_{j \in R\left(t_{i}\right)} X_{j} \exp \left(X_{j}^{\prime}(t) \hat{\beta}\right)}{\sum_{j \in R\left(t_{i}\right)} \exp \left(X_{j}^{\prime}(t) \hat{\beta}\right)}\right]=0
$$

Na prática, pode haver coincidência entre tempos de falhas e entre tempos de falha e de censura. No segundo caso, pode-se considerar que a censura ocorra após a falha. A função de máxima verossimilhança parcial precisa ser modificada, conforme sugerido por Breslow (1972) e Peto (1972). As propriedades assintóticas da função de máxima verossimilhança parcial foram derivadas por Andersen e Gill (1982), que mostraram como construir intervalos de confiança e realizar testes de hipóteses sobre os parâmetros estimados. 
Com o objetivo de verificar a qualidade do ajustamento, o percentual de previsões corretas feitas pelo modelo estimado pode ser avaliado. É baseado na soma de dois termos:

- Percentual de casos que foram previstos como inadimplentes em dado período e que atingiram essa condição nos dados observados;

- Percentual de casos que foram previstos como adimplentes em dado período e que foram adimplentes nos dados observados.

Quanto maior for a taxa de sucesso, melhor o ajustamento do modelo aos dados.

Outra forma de se verificar a qualidade do ajustamento é por meio dos erros tipo I e tipo II. Esse procedimento será aplicado sob a hipótese de que o tomador de empréstimo irá inadimplir depois de dado período, $\alpha$, definido a partir da matriz de migração.

\section{Resultados}

\subsection{Dados}

O banco de dados depurado é composto por 637.704 contratos individuais de crédito imobiliário de um grande banco brasileiro, que não será revelado por motivos de confidencialidade. Esse banco responde por cerca de $75 \%$ das operações de crédito imobiliário realizadas no país. Dessa forma, ainda que se refira a apenas uma instituição financeira, o banco de dados usado na análise empírica possui ampla representatividade no mercado de empréstimos imobiliários. As operações possuem taxas de juros pós-fixadas, são contratadas com exigência de garantia real (colateral), e previsão de punição em caso de default. A punição é caracterizada por restrições a novas contratações de crédito, inclusão do nome do devedor em serviços de proteção ao crédito de acesso público e subtração do colateral em caso de inadimplência do tomador de crédito. Para a modelagem, foram consideradas operações realizadas entre janeiro de 1997 e agosto de 2008, com acompanhamento mensal do comportamento 
de pagamento até agosto de 2009 para garantir que todos os contratos tenham, no mínimo, 12 meses de análise do comportamento de pagamento.

A base de dados possui informações referentes à idade, renda mensal, estado civil e sexo dos tomadores de crédito. Os dados contratuais referem-se ao valor financiado, unidade da federação que originou o crédito, cota de financiamento, taxa de juros contratual do empréstimo, tipo de garantia (real ou financeira) e valor da garantia. A variável cota de financiamento (equity) foi quantificada por meio da razão entre o valor do financiamento e o valor da garantia. Foi realizada uma depuração do banco de dados com a exclusão de contratos que apresentaram informações inconsistentes, como campo sem informação, data inválida, renda mensal e idade com valores absurdos. Cabe ressaltar que, ao final desse processo de depuração, permaneceu o total de 637.704 observações que constituíram uma amostra não balanceada usada nas estimações.

O primeiro ponto a ser observado é que a amostra não é balanceada por causa da discrepância entre o número de contratos censurados e não censurados. Cerca de $5 \%$ das operações registradas foram classificadas como falha (default) e 95\% como censura, ou seja, não apresentam default ou deixaram de ser observadas durante o período. Em razão da disparidade identificada entre as quantidades dos contratos censurados e não censurados, foram realizadas duas estimações, ambas usando a amostra depurada. A primeira seguiu Whalen (1991), utilizando uma amostra balanceada composta por todos os contratos que atingiram default no período e o complemento estratificado de forma aleatória do subconjunto de dados censurados. Já a segunda considerou toda amostra disponível, baseando a escolha do procedimento de amostragem na disponibilidade dos dados.

Para estimar a probabilidade de default de cada contrato ao longo do seu tempo de vida, foi utilizada a regressão de Cox. O tempo de sobrevivência corresponde ao número de meses que o contrato leva para atingir 180 dias de atraso desde a sua contratação, conforme norma interna da instituição detentora da base de dados. Os contratos que não atingiram esse nível de atraso no período analisado foram considerados censurados. Nesse caso, o tempo de sobrevivência é definido como a quantidade de meses em que foram observados, tendo como limite o mês de agosto de 2009. 
As variáveis macroeconômicas dependentes do tempo incluídas na análise foram a taxa de juros real da economia, taxa de desemprego, produção industrial, taxa de inflação medida pelo IPCA e crescimento real do produto interno bruto (PIB). Para manter a compatibilidade com a amostra de contratos de crédito imobiliário, os dados são mensais no período de janeiro de 1997 e agosto de 2009. Foram criadas 27 variáveis dummies para representar cada uma das 27 unidades da federação em que o crédito foi concedido. As séries macroeconômicas foram obtidas no Instituto Brasileiro de Geografia e Estatística (IBGE), Ipeadata e Banco Central do Brasil.

\subsection{Estimação e Análise}

O modelo de risco proporcional de Cox com covariáveis dependentes do tempo foi aplicado para estimar a probabilidade de default dos contratos de empréstimos imobiliários colateralizados. Visto que 5\% dos contratos apresentaram falha e $95 \%$ foram censurados, as estimativas reportadas consideram uma amostra artificialmente balanceada. Conforme já destacado, seguindo Whalen (1991), a amostra balanceada considerou todos os contratos não censurados e selecionou aleatoriamente uma amostra de tamanho equivalente dentre aqueles classificados como censurados. Pretende-se, com isso, evitar o viés provocado pela discrepância no tamanho relativo da amostra não balanceada. ${ }^{6}$

A falha, ou default do contrato, foi estabelecida a partir da matriz de migração ao atraso, construída com informação de todos os contratos disponíveis na base de dados e apresentada na Tabela 1 . O ponto de corte adotado corresponde ao prazo de 180 dias em atraso, onde se verifica que $80 \%$ das operações que atingiram este nível migram para atrasos maiores enquanto apenas $20 \%$ voltaram à situação de adimplência, regularizando parcelas em atraso. A classificação de 180 dias de atraso como corte para o default segue norma interna adotada pela instituição financeira detentora dos dados.

O tempo de sobrevivência corresponde ao tempo que o contrato levou para atingir a situação de default, 180 dias de atraso ou o não

\footnotetext{
${ }^{6}$ A estimação foi realizada também para a carteira completa e desbalanceada, composta por 20.520 contratos com falhas e 617.184 contratos censurados. Os resultados não divergiram da amostra balanceada, e estão disponíveis mediante solicitação.
} 
pagamento de 6 prestações consecutivas. Os contratos que não atingiram este período de atraso foram classificados como censurados e seu tempo de sobrevivência definido como o número de meses que foram observados.

As estimações foram conduzidas no pacote estatístico SAS, versão 9.1, que possui o modelo de Cox implementado sob o procedimento "proc PHREG". A estimação dos parâmetros deu-se por meio do método da máxima verossimilhança parcial, conforme sugerido por Cox $(1972,1975)$. A seleção de variáveis explicativas que deveriam permanecer no modelo estimado deu-se pela técnica "stepwise", onde a inclusão é feita de forma individualizada e a manutenção ou exclusão é condicionada ao nível de 5\% de significância. Finalmente, os critérios de informação de Akaike (AIC) e Schwartz (SBC) foram aplicados para a escolha do melhor modelo. A Tabela 2 reporta os resultados obtidos para a amostra balanceada.

Tabela 2 - Modelo de Risco Proporcional de Cox: Amostra Balanceada

\begin{tabular}{lcccc}
\hline Variável & Coeficiente & Valor-p & Razão de risco & Efeito sobre $Y$ \\
\hline Tx juro real econ. & $-1,5563$ & .0001 & 0,211 & $-0,7891$ \\
Tx juro real empr. & 0,1030 & .0001 & 1,108 & 0,1084 \\
Valor empréstimo & 0,0021 & .0001 & 1,002 & 0,0021 \\
Idade tomador & $-0,0349$ & .0001 & 0,966 & $-0,0343$ \\
Renda tomador & $-0,0043$ & .0001 & 0,996 & $-0,0043$ \\
Tomador solteiro & 0,0617 & .0001 & 1,064 & 0,0636 \\
Tomador casado & $-0,0628$ & .0001 & 0,939 & $-0,0609$ \\
Tipo garantia & $-2,7519$ & .0001 & 0,064 & $-0,9362$ \\
Cota financiamento & 0,6948 & .0001 & 2,003 & 1,0030 \\
Crescimento PIB & $-0,1172$ & .0001 & 0,889 & $-0,1105$ \\
Valor garantia & $-0,0647$ & .0001 & 0,937 & $-0,0626$ \\
Dummies estaduais & sim & & & \\
\hline
\end{tabular}

Nota: a variável dependente (Y) é a probabilidade de default.

A informação contida na coluna razão de risco indica o impacto, positivo ou negativo, na probabilidade (ou risco) de default do contrato, decorrente da variação de uma unidade na variável analisada, mantendo inalterado o valor das demais variáveis. Formalmente, é definida por:

$$
R R=\exp (\hat{\beta})
$$


A relação entre a probabilidade de default e as variáveis explicativas do modelo pode ser observada pelo sinal do parâmetro estimado. Já a magnitude do impacto é obtida subtraindo-se 1 da razão de risco daquela variável, conforme reportado na coluna "efeito sobre Y" da Tabela 2.

Antes de discutir os resultados empíricos, algumas condições que devem ser observadas quando da aplicação do modelo. ${ }^{7}$ A hipótese de mercados incompletos é necessária por causa da presença de default na modelagem. Caso os mercados sejam completos, todos os compromissos financeiros seriam honrados e o Teorema 2 não teria validade. Os resultados teóricos foram derivados para empréstimos com garantia (colateral) real. Desse modo, a evidência empírica não pode ser estendida para outros tipos de empréstimos, que não exija colateral ou possuam colateral financeiro e não físico.

Além disso, como se trata de um modelo em que o bem de consumo é o numerário, não é possível realizar inferência sobre preços nominais e inflação. Para manter o foco do trabalho, a estrutura financeira foi simplificada para possuir apenas 2 ativos, sendo um arriscado e outro livre de risco. Essa estrutura, contudo, pode ser generalizada para incluir outros ativos, inclusive de diferentes tipos, como aqueles que não possuem colateral, desde que sejam preservados os dois ativos iniciais.

Quanto aos coeficientes estimados, as análises apresentadas a seguir referem-se ao grupo de indivíduos que compõem a carteira de empréstimos imobiliários analisada. Dessa forma, a generalização dos resultados obtidos para outras amostras de indivíduos ou carteiras de crédito alternativas não é imediata. ${ }^{8}$ Caso isso ocorra, deve-se atribuir devida cautela à análise.

Tendo em mente as restrições anteriores, os resultados revelam que o aumento de um ponto percentual na taxa de juro real do contrato eleva em $10,84 \%$ a probabilidade de o contrato entrar em default. Este resultado ratifica a relação positiva entre taxa de juro do contrato e a probabilidade de default derivada pelo modelo teórico. Isso é economicamente justificável, pois quanto maior a taxa

\footnotetext{
${ }^{7}$ Os autores agradecem a um avaliador anônimo por sugerir a inclusão dessa discussão.

${ }^{8}$ Note que não há restrições quanto a unidades de medidas ou valores assumidos pelas variáveis explicativas porque a formulação teórica do modelo de risco proporcional de Cox garante que a razão de risco estará no intervalo $(0,1)$.
} 
de juros cobrada no empréstimo maior será o comprometimento da renda do agente e, consequentemente, menor a sua capacidade de pagamento.

A variável taxa de juro real da economia possui um sinal negativo, em conformidade com o resultado do Teorema 2. Numericamente, revela que uma queda de um ponto percentual no valor da taxa de juro real básica da economia acarreta um aumento médio na probabilidade de default da ordem de 78,91\%. A intuição econômica para esse resultado é que a redução da taxa de juro real, decorrente de política monetária expansionista, por exemplo, implica menor retorno financeiro das operações de tesouraria realizadas por instituições financeiras. Essa perda de receita é compensada por meio da expansão da carteira de crédito, viabilizada pela flexibilização das avaliações de risco. O banco passa a conceder crédito a tomadores mais arriscados, elevando a probabilidade de default na economia. ${ }^{9}$

A magnitude do efeito da queda da taxa de juro real sobre a probabilidade de default é expressiva. Contudo, uma alteração de $1 \%$ na taxa de juro real básica da economia também é bastante significativa. Em termos relativos, é de esperar que haja um amplo impacto de tal mudança sobre a economia como um todo. O consequente aumento da probabilidade de default revela um custo social importante da política monetária excessivamente expansionista. Isso não significa, porém, que a autoridade monetária deva abdicar de mudanças na taxa de juro mais adequadas à conjuntura macroeconômica. Sugere, apenas, que o custo social de tal política seja levado em consideração quando de sua edição. Ciente desse custo social, medidas compensatórias devem ser editadas para contrabalançar a resultante perda de bem-estar da sociedade.

Analogamente, o aumento de um ponto percentual na variável cota de financiamento provoca uma elevação na probabilidade de default. A cota de financiamento corresponde à razão entre valor do financiamento e valor da garantia. Assim, quanto maior for a cota, maior será o valor financiado e o consequente comprometimento da prestação em relação à renda do tomador.

\footnotetext{
${ }^{9}$ Vale ressaltar que, conforme mostram Dubey, Geanakoplos e Shubik (2005), esta estratégia não implica seleção adversa por causa do requerimento de colateral na concessão do empréstimo.
} 
Conforme esperado, há uma relação positiva entre o valor do empréstimo e probabilidade de default. Especificamente, um aumento no valor do empréstimo aumenta em cerca de $0,20 \%$ a probabilidade de default. A justificativa é que quanto maior for o valor do empréstimo, maior tende a ser o comprometimento da renda do tomador e, assim, maior a sua probabilidade de default.

A variável idade, para o conjunto de indivíduos que compõem a carteira imobiliária analisada, apresenta relação negativa com a probabilidade de default, revelando que tomadores mais velhos carregam menor possibilidade de inadimplência, sendo o impacto da ordem de $-3,43 \%$ para cada ano adicional na idade. Considera-se que os tomadores de empréstimos mais jovens, por estarem ingressando no mercado de trabalho, contam com menor experiência profissional e podem possuir menos estabilidade financeira do que os tomadores mais velhos. Logo, na referida carteira de empréstimos imobiliários, os tomadores jovens carregam uma maior probabilidade de default. ${ }^{10}$

A renda real do tomador apresentou relação negativa com a probabilidade de default, sendo o impacto da ordem de -0,43\%. Esse resultado justifica-se pelo fato de que, quanto maior for a renda, menor será o comprometimento da capacidade de pagamento do tomador, assegurando uma menor probabilidade de inadimplência.

As variáveis dummies para os estados civis solteiro e casado em relação a outros estados civis apresentaram relações positiva e negativa com a probabilidade de default, respectivamente. Os impactos foram de 6,36\% e -6,09\%, respectivamente. Intuitivamente, supõe-se que o tomador de empréstimo que é casado possui mais maturidade e comprometimento com as obrigações financeiras assumidas. Adicionalmente, tende a contar com maior estabilidade financeira do que tomadores solteiros. Tais características concorrem para reduzir sua probabilidade de default em relação a tomadores que possuem outros estados civis.

O coeficiente estimado para a variável "tipo de garantia" apresentou sinal negativo, com um impacto $-93,62 \%$ sobre a probabilidade de default. Desse modo, quando a garantia oferecida é um bem real, a

\footnotetext{
${ }^{10}$ Vale ressaltar que tanto esse quanto os demais resultados empíricos obtidos aplicam-se à carteira de empréstimos imobiliários analisada. Não se pretende, com a análise apresentada, generalizar as interpretações para outras carteiras de crédito ou distintas amostras de indivíduos.
} 
probabilidade de default é menor do que quando a garantia do contrato é financeira, representada por seguro ou caução de depósito. No caso de garantia real, a penalidade ao tomador na ocorrência de default é a perda do bem financiado (imóvel), cuja propriedade é transferida para o credor, que pode incorporá-lo ao seu patrimônio ou vendê-lo para amortizar a dívida.

As variáveis crescimento real do PIB e valor da garantia apresentaram sinais negativos, indicando que contribuem para reduzir a probabilidade de default. Os efeitos estimados foram de $-11,05 \%$ e $-6,26 \%$ para cada uma daquelas variáveis, respectivamente. O crescimento do PIB indica que a economia está aquecida, com mais emprego e maior nível de renda, elementos que contribuem para reduzir a probabilidade de default. O valor da garantia, por sua vez, deve satisfazer a restrição de ser igual ou superior ao empréstimo. Quanto maior for a diferença em relação ao empréstimo adquirido, menor será a probabilidade de default porque a inadimplência acarretará a perda do colateral.

Para verificar a qualidade do ajustamento, foram comparadas as previsões feitas fora da amostra para as probabilidades de default com a taxa de default efetiva observada nos dados. Para tanto, foram selecionados todos os contratos com tempo de sobrevivência igual a doze meses. Aqueles contratos que apresentaram probabilidade de sobrevivência inferior a $65 \%$ foram classificados como passíveis de default no $12^{\circ}$ mês. Compararam-se, então, as previsões do modelo com os contratos que efetivamente entraram em default no $12^{\circ}$ mês de vida. A razão identificada entre a quantidade de contratos projetados como default e a quantidade de contratos que, de fato, entraram em default foi de 0,7562 . Isso indica que $75,62 \%$ das ocorrências de default naquele grupo de contratos foram corretamente projetadas, indicando um bom desempenho para o modelo estimado. ${ }^{11}$

É possível, também, identificar os erros tipo I e tipo II incorridos nas projeções. O erro tipo I, que corresponde a classificar como não default aqueles contratos que acabaram atingindo default no $12^{\circ}$ mês, foi de $22,83 \%$. Isto indica que dos contratos que se esperava não entrarem em default até o $12^{\circ}$ mês, $22,83 \%$ entraram. Já o erro tipo II, que corresponde a classificar como default os contratos que não

\footnotetext{
${ }^{11}$ Rocha (1999), por exemplo, considera que 40\% de acerto representa uma precisão de classificação relativamente alta.
} 
atingiram essa condição, foi de 30,16\%. Isso significa que dentre os contratos que se esperavam entrar em default no $12^{\circ}$ mês, $30,16 \%$ não atingiram essa condição. Vale ressaltar que, no processo de gestão de risco de crédito, o erro tipo II é mais aceitável por ser conservador. Além disso, aqueles contratos podem se tornar inadimplentes em horizontes superiores a 12 meses.

\section{Conclusão}

Este artigo investigou, teórica e empiricamente, a relação entre taxas de juros e probabilidade de default. O modelo teórico, baseado em Dubey, Geanakoplos e Zame (1995), caracteriza-se por equilíbrio geral com mercados incompletos, exigência de colateral real e punição aos indivíduos em caso de default. Observadas essas condições, além da existência de uma estrutura financeira que inclua ativo arriscado e ativo sem risco, estabelecidas para a construção do modelo, foi encontrada uma relação positiva entre probabilidade de default e a taxa de juro real do empréstimo e negativa entre a probabilidade de default e taxa de juro real básica da economia. Esses resultados foram testados empiricamente por meio da aplicação do modelo de risco proporcional de Cox com variáveis dependentes do tempo. A amostra, obtida junto a um grande banco brasileiro, contemplou 637.704 empréstimos colateralizados de uma carteira imobiliária e cobriu o período de janeiro de 1997 a agosto de 2009.

Para o conjunto de indivíduos que compõem a carteira de empréstimos imobiliários analisada, os resultados empíricos confirmam os sinais encontrados pelo modelo teórico para a relação entre taxas de juros e probabilidade de default. Intuitivamente, uma redução na taxa de juros real da economia decorrente de uma política monetária expansionista, por exemplo, provoca uma queda no retorno financeiro de operações de tesouraria realizadas pela instituição financeira. Para compensar essa perda, os bancos expandem suas carteiras de crédito, flexibilizando as análises de avaliação de risco dos tomadores. Com isso, trazem para o mercado indivíduos que foram classificados como muito arriscados no passado, o que resulta em elevação da probabilidade de default. Isso ocorre, especialmente, em períodos de crise econômica, como aquela que atingiu a economia americana no final de 2007 e se espalhou por diversos países do mundo. As medidas expansionistas adotadas por diversos bancos centrais, in- 
cluindo o Banco Central do Brasil, podem resultar em um aumento na probabilidade de default na economia.

Por outro lado, uma elevação na taxa de juro real do empréstimo aumenta a probabilidade de default porque reduz a capacidade de pagamento do tomador. Isso faz com que o custo do empréstimo se torne mais alto e, assim, aumente sua probabilidade de inadimplência.

Outros resultados interessantes obtidos pelo modelo empírico foram as relações negativas entre idade e tipo de garantia e a probabilidade de default. Assim, tomadores mais velhos carregam menores probabilidades de default, sendo o impacto da ordem de $-3,43 \%$ para cada ano adicional na idade. Quando a garantia oferecida é um imóvel, a probabilidade de default é menor em $93,62 \%$ do que quando a garantia do contrato é financeira.

A renda real do tomador e o crescimento da economia também impactam negativamente a probabilidade de default. O primeiro resultado deve-se ao fato de que, quanto maior for a renda, menor será o comprometimento da capacidade de pagamento do tomador, assegurando sua solvência no mercado. Já o crescimento do PIB mostra que a economia está aquecida, com as pessoas empregadas e aumentando seus rendimentos. Esses elementos conduzem a uma menor probabilidade de default.

Vale ressaltar que não se pretende condenar o uso de políticas monetárias expansionistas para combater recessões econômicas. Pretendese, apenas, advertir que tais políticas carregam custos sociais importantes, sendo um deles a elevação da probabilidade de default dos indivíduos na economia. Esse efeito adverso deve ser levado em consideração quando da elaboração e análise dos custos sociais de políticas econômicas contracíclicas.

\section{Referências}

Ali, A., Daly, K. (2010) Macroeconomic determinants of credit risk: recent evidence from a cross Country study. International Review of Financial Analysis, v. 19, p. 165-171.

Andreeva, G. (2006) European generic scoring models using survival analysis. Journal of the Operational Research Society, v. 57, p. 1180-1187. 
Banasik, J., Crook, J., Thomas, L. (1999) Not if but when will borrowers default. Journal of the Operational Research Society, v. 50, p. 1185-1190.

Barth, J., Gotur, P., Neela, M., Yezer, A. (1983) The effect of government regulations of Personal Loans Markets: A Tobit Estimation on Microeconomic Model. Journal of Finance, v. 38, p. 1233-1251.

Bellotti, T., Crook, J. (2007) Credit Scoring With Macroeconomic Variables Using Survival Analysis. University of Edinburgh Working Paper.

Breslow, N. (1972) Contribuição à discussão do artigo de D. R. Cox. Journal of the Royal Statistical Society B, v. 34, p. 216-217.

Carling, K., Jacobson, T., Linde, J., Roszbach, K. (2007) Corporate Credit Risk Modeling and the Macroeconomy. Journal of Banking and Finance, v. 31, p. 845-868.

Chu, V. (2001) Principais Fatores Macroeconômicos da Inadimplência Bancária no Brasil. Mimeo. Banco Central do Brasil.

Cox, (1972) Regression Models and Life Tables (with discussion). Journal of the Royal Statistical Society B, v. 34, p. 187-220.

Cox, (1975) Partial Likelihood. Biometrika, v. 62, p. 269-276.

Divino, J. A., Lima, E., Orrillo, J. (2010) Interest Rates and Default in Unsecured Loan Markets. 2010 World Congress of the Econometric Society.

Dubey, P., Geanakoplos, J., Shubik, M. (1990) Default and Efficiency in a General Equilibrium Model with Incomplete Markets. Cowles Foundation Discussion Paper n ${ }^{\circ} 773 R$.

Dubey, P., Geanakoplos, J., Zame, W. (1995) Default, Collateral and Derivatives. Mimeo. Yale University.

Dubey, P., Geanakoplos, J., Shubik, M. (2005) Default and Punishment in General Equilibrium. Econometrica, v. 73, p. 1-37.

Lin, E., White, M. (2001) Bankruptcy and the Market Mortgage and Home Improvement Loans. Journal of Urban Economics, v. 50, p. 138-162.

Magill, M., Quinzii, M. (1996) Theory of Incomplete Markets. Cambridge: The MIT Press. 540 p.

Martins, M. S., Galli, O. C. (2007) A previsão de insolvência pelo modelo de Cox: Uma aplicação para a análise de risco de companhias abertas brasileiras. REAd - Edição 55, v. 13 No 1.

Pascoa, M. R., Seghir, A. (2009) Harsh default penalties lead to Ponzi schemes. Games and Economic Behavior, v. 65, p. 270-286.

Peto, R. (1972) Discussion of the paper by D. R. Cox. Journal of the Royal Statistical Society B, v. 34, p. 205-207.

Rocha, F. (1999) Previsão de Falência Bancária: um Modelo de Risco Proporcional. Pesquisa e Planejamento Econômico, v. 29, p. 137-152.

Stepanova, M., Thomas, L. (2001) PHAB Scores: Proportional Hazards Analysis Behavioural Scores. Journal of the Operational Research Society, v. 52, p. 1007-1016.

Stepanova, M., Thomas, L. (2002) Survival Analysis Methods for Personal Loan Data. Operations Research, v. 50, p. 277-289.

Tang, L., Thomas, L., Thomas, S., Bozzetto, J-F. (2007) It's the Economy Stupid: Modelling Financial Product Purchases. International Journal of Bank Marketing, v. 25, p. 22-38.

Whalen, G. (1991) A Proportional Hazards Model of Bank Failure: An Examination of its Usefulness as an Early Warning Tool. Economic Review of the Federal Reserve Bank of Cleveland, v. 27, p. 21-31.

Zame, W. (1993) Efficiency and the Role of Default when Security Markets are Incomplete. American Economic Review, v. 83, p. 1142-1164. 


\section{Anexo}

A prova do Teorema 2 é apresentada a seguir. Se existe equilíbrio, então o problema de cada agente $h$ tem solução, de maneira que as condições de Kuhn-Tucker são necessárias. Mais precisamente, temos que o Langrangeano é dado por:

$$
\begin{aligned}
& L(x, \theta, \varphi, b, D, \alpha, \beta)=u^{h}\left(x_{o}+C \varphi, \tilde{x}\right)-\sum_{s} \lambda_{s}\left[r_{s} \varphi-D_{s}\right]^{+}-\alpha_{o}\left(x_{o}+q b+\pi \theta+C \varphi-\omega_{o}^{h}-\pi \phi\right) \\
& -\sum_{s} \alpha_{s}\left(x_{s}+D_{s}-\omega_{s}^{h}-t_{s} r_{s} \theta-(1+r) b-C_{s} \varphi\right)-\sum_{s} \beta_{s}\left(\min \left\{r_{s}, C_{s}\right\} \varphi-D\right)
\end{aligned}
$$

As condições necessárias de Kuhn-Tucker implicam que há multiplicadores $\alpha \in R_{+}^{1+S}$ e $\beta \in R_{+}^{S}$ e super gradientes $\left(\lambda_{s} g_{s}\right)$ da penalidade com respeito à inadimplência, com $g_{s} \in[0,1]$ e $g_{s}=1$ se a inadimplência ocorre, tais que:

1. $\frac{\partial u^{h}\left(\tilde{x}_{o}^{h},\left(x_{s}\right)\right)}{\partial x_{o}} \leq \alpha_{o}$, com igualdade se $x_{o}>0$

2. $\frac{\partial u^{h}\left(\tilde{x}_{o}^{h},\left(x_{s}\right)\right)}{\partial x_{s}} \leq \alpha_{s}$, com igualdade se $x_{s}>0, \forall s \in \mathcal{S}$

3. $\frac{\partial u^{h}\left(\tilde{x}_{o}^{h},\left(x_{s}\right)\right)}{\partial x_{o}} C-\sum_{s} \lambda_{s} r_{s} g_{s}-\alpha_{o}(C-\pi)+\sum_{s} \alpha_{s} C_{s}-\sum_{s} \beta_{s} \min \left\{r_{s}, C_{s}\right\} \leq 0$,

com igualdade se $\varphi>0$

4. $-\alpha_{o} \pi+\sum_{s} \alpha_{s} t_{s} r_{s} \leq 0$, com igualdade se $\theta>0$

5. $-\alpha_{o} q+\sum_{s} \alpha_{s}(1+r)=0$

6. $\lambda_{s} g_{s}-\alpha_{s}+\beta_{s} \leq 0$, com igualdade se $D_{s}>0$ e $g_{s}=1$ se $D_{s}<r_{s} \varphi$ e $g_{s} \in[0,1]$ caso contrário.

Além disso, tem-se que:

$$
\begin{aligned}
& \alpha_{0}\left[x_{0}+q b+\pi \theta+C \varphi-\omega_{0}^{h}-\pi \varphi\right]=0 \\
& \alpha_{s}\left[x_{s}+D_{s}-\omega_{s}^{h}-t_{s} r_{s} \theta-(1+r) b-C_{s} \varphi\right]=0, s \in \mathcal{S} \\
& \beta_{s}\left[\min \left\{r_{s}, C_{s}\right\} \varphi-D_{s}\right], \quad s \in \mathcal{S}
\end{aligned}
$$


Desde que $\min \left\{r_{s}, C_{s}\right\}>0$, então a Equação (4) do problema do consumidor implica que $D_{s}>0$. Assim, pelo item 6, segue-se que:

$$
\begin{aligned}
& \lambda_{s} g_{s}+\beta_{s}=\alpha_{s} \\
& D_{s}>\min \left\{r_{s}, C_{s}\right\} \varphi \Rightarrow \beta_{s}=0 \Rightarrow \lambda_{s} g_{s}=\alpha_{s} \leq \lambda_{s} .
\end{aligned}
$$

Portanto,

$$
\lambda_{s}<\alpha_{s} \Rightarrow D_{s}=\min \left\{r_{s}, C_{s}\right\} \varphi .
$$

Dessa maneira, a inadimplência é máxima.

Por outro lado, se $\varphi>0$ e $D_{s}<r_{s} \varphi$ então existe inadimplência de maneira que $g_{s}=1$. Desse modo, se $\left.D_{s} \in\right] \min \left\{r_{s}, C_{s}\right\} \varphi, r_{s} \varphi[$ então $\lambda_{s}=\alpha_{s}$ e $\beta_{s}=0$.

Assumindo que haja inadimplência que não é máxima e $(x, \theta, \varphi)>>0$, tem-se que os itens (1), (2), e (3) implicam:

$$
\alpha_{o} C-\sum_{s} \lambda_{s} r_{s}-\alpha_{o}(C-\pi)+\sum_{s} \alpha_{s} C_{s}=0
$$

Combinando (A.4) com os itens (4) e (5) e assumindo $\lambda_{s}=\lambda, \forall s$, tem-se:

$$
S R=\sum_{s} t_{s} R_{s}=\sum_{s}\left(R_{s}-\tilde{C}_{s}\right)
$$

em que

$$
R=\frac{(1+r)}{q}, R_{s}=\frac{r_{s}}{\pi} \mathrm{e} \sum_{s} k_{s} R_{s}=\sum_{s} \tilde{C}_{s}
$$

sendo $k_{s}=1-t_{s}, \forall s$.

$$
\sum_{s} t_{s} R_{s}=S R
$$

Defina a função $F:[0,1]^{S} \times R_{+}^{S} \times R \rightarrow R$ como sendo

$$
F\left(\left(t_{s}\right),\left(R_{s}\right), R\right)=\sum_{s} t_{s} R_{s}-S R
$$

Derivando (A.9) em relação a $t_{s}$ tem-se:

$$
\frac{\partial F}{\partial t_{s}}=R_{s}>0 .
$$


Portanto, podemos aplicar o teorema da função implícita, dado que (A.8) é sempre satisfeita, e assim obter:

$$
\begin{gathered}
\frac{\partial t_{s}}{\partial R_{s}}=-\frac{\frac{\partial F}{\partial R_{s}}}{\frac{\partial F}{\partial t_{s}}}=-\frac{t_{s}}{R_{s}}, s \in \mathcal{S} \\
\frac{\partial t_{s}}{\partial R}=-\frac{\frac{\partial F}{\partial R}}{\frac{\partial F}{\partial t_{s}}}=-\frac{-S}{R_{s}}
\end{gathered}
$$

Definindo a taxa de inadimplência como sendo 1 menos a taxa de pagamento, resulta:

$$
k_{s}=1-t_{s}, s \in \mathcal{S}
$$

Logo, a partir de (A.10) e (A.11), tem-se que:

$$
\frac{\partial k_{s}}{\partial R_{s}} \geq 0 \text { and } \frac{\partial k_{s}}{\partial R}<0,
$$

o que completa a prova. 